\title{
Markov modelling on the effectiveness of sanctions: A case study of the Falklands War
}

\author{
$\begin{array}{ll}\text { A. H. Pincombe } & \text { B. M. Pincombe } \\ & 2\end{array}$
}

(Received 11 August 2006; revised 7 November 2007)

\begin{abstract}
Within a war fighting scenario, high level effects will emerge as a result of actions or constraints at a lower level. Simulation of this emergence for realistic numbers of components has not been possible because of the large number of system states to be considered. We use a previously developed envelope method and show that it enables a dramatic reduction in the number of states that must be considered, as long as the optimal attack sequence is known. The method is applied to an example (for which the optimal attack sequence is obvious) from the Falklands War, to evaluate the effectiveness of a particular arms sanction. The outcome demonstrates that the envelope method offers an achievable data preparation load as well as computational tractability and that it is a practical way to evaluate the benefits of particular sanctions when the optimal sequence is known.
\end{abstract}

See http://anziamj.austms.org.au/ojs/index.php/ANZIAMJ/article/view/80 for this article, (c) Austral. Mathematical Soc. 2007. Published November 14, 2007. ISSN 1446-8735 


\section{Contents}

1 Introduction

C528

2 Case study: The Falklands War

C529

3 Method

C530

3.1 Model . . . . . . . . . . . . . . . . . . . . C531

3.2 Assessment method . . . . . . . . . . . . . . C534

4 Results

C534

5 Discussion

C537

6 Conclusions

C539

References

C539

\section{Introduction}

The use of diplomatic pressure to obtain arms sanctions can be a highly successful way to gain an advantage during a war. Typically, a broad sanctions regime is sought but this is not always either achievable or practically enforcable. Where it is impossible to achieve a total sanctions regime diplomatic pressure would ideally be targeted at those sanctions that are most likely to make a difference to the outcome of the war. But how can we decide a priori about the advantage that could be gained from particular sanctions?

In military analysis much effort goes into the modelling of missions, where a mission is a sequence of battles in pursuit of an ultimate objective. Missions are characterised by randomness so it makes sense to place victory (the achievement of the objective) in a framework of probability. Mostly the framework is a pair of Markov models, where the states are defined by sets of 
surviving assets, with the unknown enemy intent being dealt with by game theoretic optimisation $[2,6]$. Such models are successful for missions with small numbers of interacting elements, but for more elements they become computationally intractable because of the large number of states that need to be considered. There is also a practical problem. A large number of states means an even larger number of transition probabilities that may need to be calculated by an analyst.

We have previously shown $[3,4]$ that, in some cases, the number of states can be reduced dramatically. Firstly, if we allow unrestricted replacement of disabled attack assets we can express the problem as a single Markov model, with states defined by the surviving defenders. Instead of attackers being assessed on the basis of their likelihood of victory, they are measured, in a negative sense, by the losses they incur in achieving victory. The outcome is an estimate of the probability of victory, for the attackers, as a function of losses incurred. Secondly, when the best sequence of attacks is known a priori we can estimate the spread of possible results via an envelope comprised of the low-cost and high-cost sequences of battles, that is, where the next opponent is chosen to minimise (or maximise) the overall cost of the mission. Together, these changes make it feasible to analyse missions with realistic numbers of interacting elements.

We use a real mission from the Falklands War as a test for our method. For this mission, the low-cost and high-cost sequences of targets are obvious and the results show the very high value of the sanctions that Britain obtained from the USA over the supply of weapons to Argentina.

\section{Case study: The Falklands War}

The Falklands War has been chosen as a case study because it was well documented and recorded on both sides, was a medium size war giving enough cases to assess without overwhelming with information, happened in 1982 so 
has information available, and because arms sanctions formed an important part of the UK strategy in its prosecution. Indeed, on the first day of the war France embargoed missiles and aircraft bound for Argentina on the request of the UK [1]. The importance of French sanctions on the outcome of the war is widely recognised [5] as is the importance of sanctions implemented less enthusiastically by the US $[5,1]$. There was a strong group of Argentine supporters in the US administration and it was entirely plausible that only targeted arms sanctions would be achieved [1]. In the end the US implemented a broad sanctions regime against Argentina [1]. Amongst the sanction induced weaknesses in Argentine forces proposed as critical components in their defeat was their lack of access to high drag tail assemblies for their Mk80 series dumb-bombs. To prevent accidental detonation near the aircraft, fuses take some time to arm. For low level attacks, a high drag tail assembly (ballute) was needed to slow the bomb sufficiently for the fuse to consistently arm prior to impact. All Royal Navy (RN) vessels hit by bombs that detonated were unable to continue their duties [1]. Seven major $\mathrm{RN}$ vessels had bombs lodge in them but not detonate [1]. Five of these vessels contributed significantly to air defence and three of these were hit by multiple bombs that did not detonate [1].

\section{Method}

A sanction to stop supply of bomb ballutes will change the conditional probability $p_{d}$ that a bomb will explode, given that it hits the target. We can obtain estimates of $p_{d}$ both with, and without, sanctions, but what will the associated bombers be doing and what is their likelihood of success?

If we use the context of the Falklands War and look at the question of sanctions from the British viewpoint, we know that the Naval force is central to the prosecution of the war and that the force needs to remain within contact distance of the Falklands. We also know that several layers of defence 
are to be used and that withdrawal of any of the layers would compromise the conduct of the war and we know the threshold value of losses or damage that would bring about the withdrawal of a defence layer.

Damage to a defence layer might be inflicted in a variety of ways, for example by missiles as well as by bombing. However, the effects of bombing do not depend on the use of missiles, or on any other attack methods, thus bombing can be analysed independently. Other attack methods might also need to be analysed to determine the need for other sanctions, but these are duplicate problems to the one considered here.

We also know the likelihood of a layer being reinforced, and we argue that reinforcement is a confounding factor that can be considered separately. We represent its effect here via sensitivity analysis.

\subsection{Model}

A Markov process is based on a state space and, in the case considered here, the number of states is finite with the states representing combinations of defence assets. Assets may be of different types and all assets that interact with each other need to be included. There is a vector of initial state probabilities $\mathbf{s}_{0}=\left(s_{01}, s_{02}, \ldots, s_{0 n}\right)$, where $s_{0 i}$ is the probability that the process is initially in state $i$, and there is a matrix $P$ of transition probabilities with elements

$$
p_{i j}=\operatorname{Pr}\left[X_{k+1}=j \mid X_{k}=i\right],
$$

where $X_{k}$ is the state the process is in at time step $k$. For the case analysed here

$$
\mathbf{s}_{0}=(0,0, \ldots, 1),
$$

and the transition matrix $P$ is bidiagonal in these models because there is no replacement and the state moves monotonically from $X=n$ to $X=1$. The rows of the transition matrix each sum to one and the vector of state 
probabilities at time step $k$ is defined in the usual way,

$$
\mathbf{s}_{k}=\mathbf{s}_{0} P^{k},
$$

where $\mathbf{s}_{k}=\left(s_{k 1}, s_{k 2}, \ldots, s_{k n}\right)$. Once the system is in state $X=i$, it will either stay in that state (probability $=p_{i i}$ ), or leave it. In these models, each state is visited once.

Some special features of these models need to be explained including how the attack assets are isolated from the state, how the envelope is formed, and what it means for the computational load, the role of tactics, and the generalised concept of time step and its consequences.

A simple change of objective enables us to exclude the attack assets from the state. Instead of asking which of two adversaries will win a battle, we seek the number and types of casualties that the attacker will suffer in achieving victory. This is equivalent to allowing the attacker to have unlimited replacements. The cost of victory in each case is arguably as useful as a record of wins and losses would be.

In simple cases, the envelope is formed by the upper and lower bounds for costs. This may create a multi-criteria decision problem in more complex cases but there is room to move since the envelope just has to be indicative of high and low costs and does not need to be formed from extremals. Once all other factors have been set, the cost of victory will depend on the order in which the targets are attacked, so a very large number of states might need to be considered. By setting up a model for a single sequence of single targets, the number of states required is less than or equal to the number of defence assets. Thus a small Markov model is set up for each of the bounds. In many cases the high and low cost sequences are far from obvious, but in the case of the Falklands air war the sequences are obvious.

The probabilities for transitions from each state to the next state, in the sequence of states that defines a particular bound, are determined from the capabilities of the attackers, including the detonation probability $p_{d}$, 
and those of the remaining defenders as well as on the tactics used. This information would come from subject matter experts. The tactics that are used may include the use of multiple groupings of aircraft, with each group targeting a different ship.

Since a time step in a Markov model is completely unencumbered, we regard the attack of each group of aircraft, with its single target, as occuring at a different time step, as long as we take account of the coordinated nature of the group attacks when we calculate the transition probabilities. Thus, any real case of a multi-target attack is viewed as a sequence of single target attacks. Such real cases are likely to produce non-optimal sequences of ship removal and their costs will thus fall within the envelope that we calculate. We use data from public records in lieu of expert advice [5].

We consider attacks by groups of four Skyhawk bombers, flying low above the sea in order to avoid detection by ship radar systems. The expected losses of each asset type incurred by an attempt to change the state become costs in the model and these are calculated along with transition probabilities. The costs associated with an attempt to leave state $X=i$, using our chosen tactics and attack capabilities, are represented by $C(i)$. In the general model $[3,4]$ the costs are more complicated, but here we deal with only a single type of attack asset and a single tactical approach. Note that there are no costs associated with state 1 (the goal state) and that the cost function (4) is strictly only correct when an attack is unsuccessful. If an attack is successful the attackers will face a reduced threat on the way back to base.

At time step $k$, the expected value for the cost is

$$
E_{k}=\mathbf{s}_{k} \cdot \mathbf{C},
$$

whereas the accumulated value of the expected cost, up to time step $K$, is

$$
T_{K}=\sum_{k=1}^{K} E_{k} .
$$




\section{$3.2 \quad$ Assessment method}

For the example, we set the threshold for withdrawal as the loss of eight ships from a defence layer and we estimate the probability of achieving this objective, via bombers alone, for each time step (where a time step is just a way to chronologically order the actions). Our model is set up from the British viewpoint so we do not set a loss threshold for bombers, merely calculating expected losses. We estimate the probability of the bombers achieving their objective, both with sanctions and without.

We use a defence layer of fourteen ships of different types and different defence capabilities, resulting in a need for more than 8000 Markov states if we were to allow for all possible sequences of states. This large number of states would be a problem. As bounds, or an envelope, for the process we use the best and worst sequences where only a single ship is targeted at each time step. This reduces the number of Markov states in our example to nine, making sensitivity analysis easy.

\section{Results}

The critical property in this analysis is the probability $p_{d}$ that a bomb will detonate once it has scored a hit. Without sanctions, that is using ballutes, this probability has a value close to 0.98 ; with sanctions, from publicly available data [5] the value would be close to 0.2 but the range of outcomes is too large to compare these two probabilities on a single graph so we use intermediate values. Figure 1 shows aircraft losses for $p_{d}=0.9$ (red), 0.6 (green), 0.3 (blue) for the low-cost order (full lines) and the high-cost order (dashed lines). For the low-cost order, when $p_{d}=0.9$ the objective would be substantially achieved for an expected loss of eight bombers. When $p_{d}=0.6$ there is a probability of about 0.8 that the objective would be achieved, with losses expected to be about thirteen bombers. When $p_{d}=0.3$ there is no real 


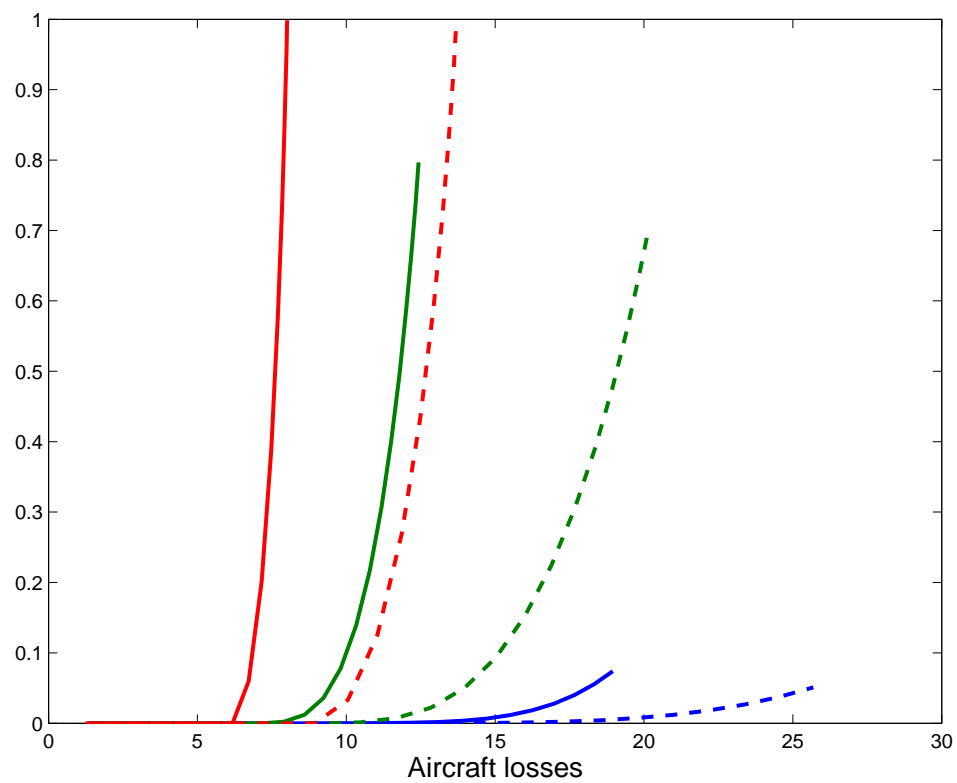

Figure 1: Comparison of three levels of probability of bombs exploding, for the two envelope cases (best and worst).

liklihood of achieving the objective. In this and other figures the results are presented after twenty timesteps, which is quite sufficient in this analysis. For the high-cost order of attack, when $p_{d}=0.9$ the objective is almost achieved in twenty time steps, for an expected loss of fourteen bombers. If $p_{d}=0.6$ there would be a probability of 0.7 of achieving the objective in twenty time steps, with an expected loss of in excess of twenty bombers. When $p_{d}=0.3$ there is no real liklihood of achieving the objective.

The above results were based on some probabilities that were deduced in part from published data for the Falklands air war and we demonstrate that these values are consistent with the actual outcome of the war. In the actual conflict, three ships were put out of action by the bombers, at a cost of nineteen bombers. Figure 2 shows the probability of being in the state 


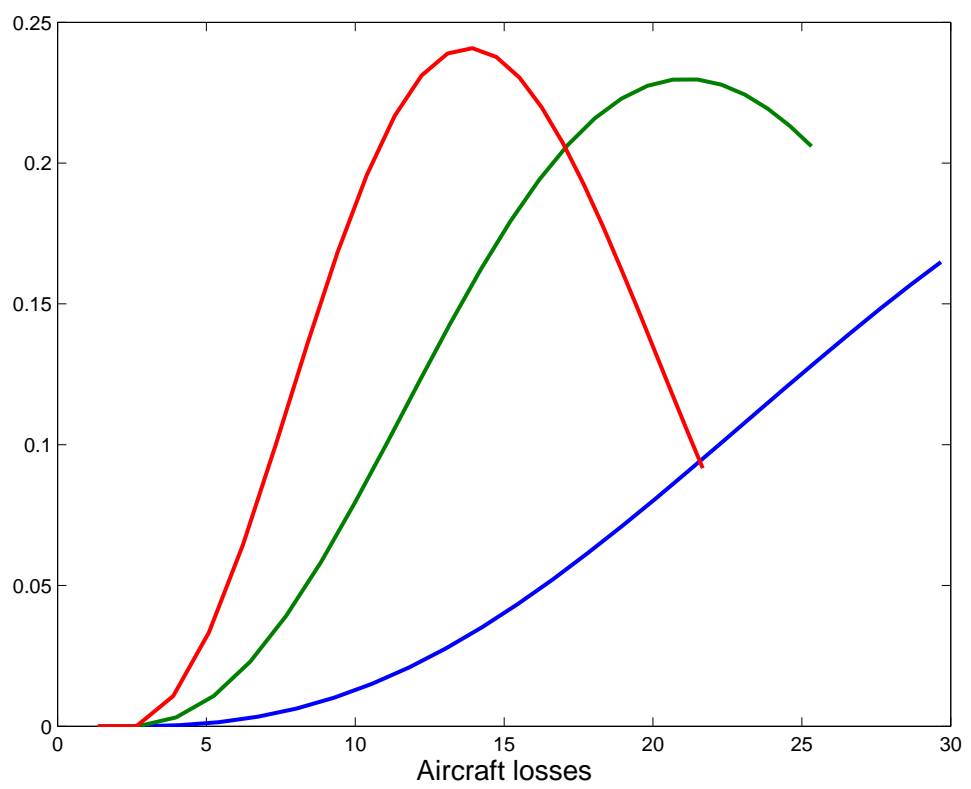

Figure 2: Probability of being in a state where three ships have been sunk, graphed against timestep.

where the three most capable ships have been eliminated, graphed against the expected loss of bombers. When $p_{d}=0.3$ (red), the highest liklihood of being in that state is associated with a loss of fourteen bombers. When $p_{d}=0.2$ (green), the highest liklihood of being in the state is associated with a loss of twenty one bombers, and when $p_{d}=0.1$ (blue), the state would be associated with greater losses.

Before the war, planners would have expected better performance from anti-aircraft defences. In some cases, these systems failed at critical times, so the actual performance is lower than the designed performance for the systems. We cater for this by looking at the case where the defence systems were thought to be four times as lethal as they turned out to be. Figure 3 represents the low-cost case by solid lines and the high-cost case by dashed 


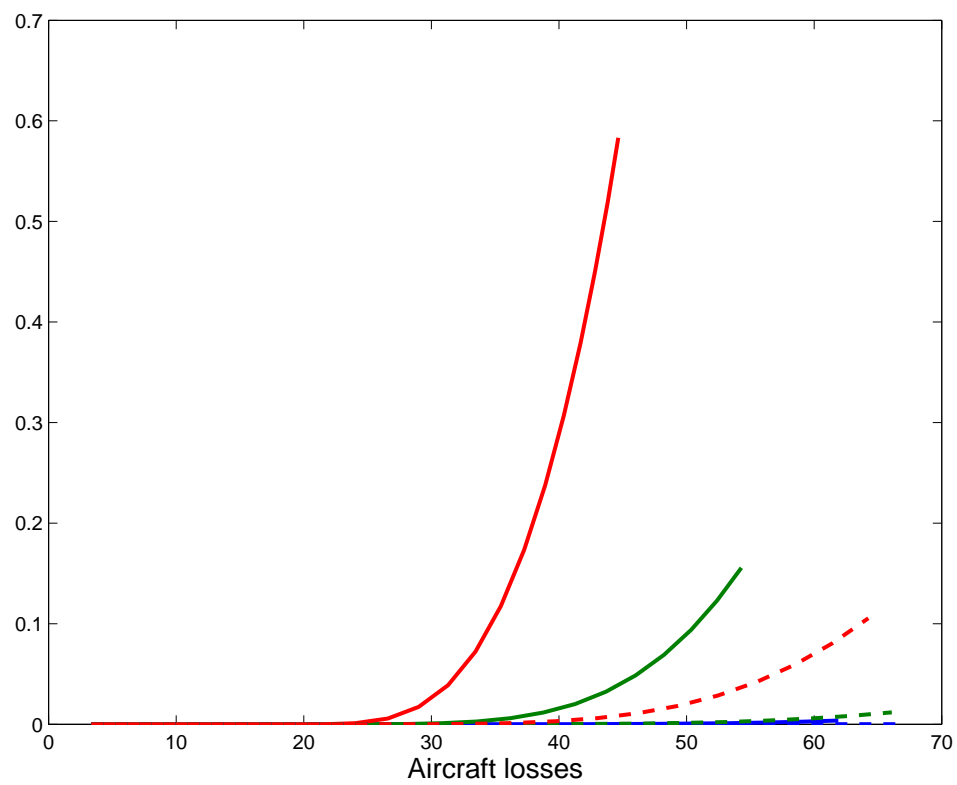

Figure 3: Comparison of three levels of probability of bombs exploding, for the two envelope cases (best and worst), for the case where ships are four times as lethal.

lines. In the low cost case for $p_{d}=0.9$, after twenty time steps the probability of achieving the objective is about 0.6 for an expected loss of about forty two bombers.

\section{Discussion}

We aimed to show that military planners could use this methodology to investigate many cases for sanctions, given that the planners would have just a few weeks to complete the investigation. We do not need to prove that sanctions were a critical factor in the course of the conflict. This result is 
well known, and the results we got in using the Markov modelling approach are thoroughly in keeping with established facts. If the sanctions on the supply of bombs and components had not been agreed with the United States, the war might have been prolonged and more costly, both in human and equipment terms. We focused on applying the method to an assessment of the effect of sanctions on the lethality of bombers and on the contributions they are likely to make both with and without sanctions. Sanctions were also achieved against other weapons, such as missiles, and these sanctions were also critical, but we can see that, without the sanctions on bombs, that is with a conditional probability of detonation of 0.98 , bombers alone could have caused a significant setback for the British forces. This result would still have been evident even if planners expected the performance of British anti-aircraft defences to be considerably better than they turned out to be.

The ability to rapidly extract the information about the effectiveness of sanctions was enabled by a very large reduction in the number of states that needed to be considered. Run times for these models were extemely short but this is not the factor that makes the method useful. Before this analysis could be done, planners would need to identify the opportunity and then calculate the transition probabilities for each of the states that would be involved. This method has made it possible to derive all of the transition probabilities in the short time that would be available, simply by a big reduction in the number of states.

We were able to calculate the transition probabilities within two days, including the time spent finding appropriate references and the time extracting data from those references. This was possible because we had previously developed much of the modelling approach, so we were familiar with it. We make no claim that the methodology, in its current form, could be used by strategic planners to achieve such timelines, merely that such results appear to be achievable with appropriate effort in implementation. 


\section{Conclusions}

The US implemented broad brush sanctions against Argentina but there was a strong chance that targeted sanctions may have been all the UK could achieve. Had this been so, sanctions against the supply of ballutes would have been identified as critical, using this method. This method is broadly applicable to other situations where sanctions are being sought and will indicate best and worst case scenarios. It is fast enough to apply to many cases in the search for a politically achievable package of targeted sanctions. Future work could include the development of approximations to enable application of the method in the absence of a computer package that copes easily with matrix algebra.

\section{References}

[1] M. Hastings and S. Jenkins, The Battle for the Falklands (Pan, 1983). C530

[2] J. Jelinek, On the deployment sequence optimality in model predictive control of military operations, in Proceedings of the American Control Conference, 2002). C529

[3] A. H. Pincombe and B. M. Pincombe, A Markov decision model for tactical military engagements, in Proceedings of ASOR2001, 2001). C529, C533

[4] A. H. Pincombe and B. M. Pincombe, A Markov based method for military analysis, Bulletin of the Australian Society for Operations Research 22 (2003) . C529, C533 
[5] D. A. Rough C. R. Smith R. A. Burden, M. I. Draper and D. L. Wilton, Falklands: The air war (Arms and Armour Press, 1986). C530, C533, C534

[6] B. Fitzpatrick W. McEneany and I. Lauko, Stochastic game approach to air operations, IEEE Transactions on Aerospace and Electronic Systems 40 (2004) 1191-1216. C529 


\section{Author addresses}

1. A. H. Pincombe, Defence Science and Technology Organisation, Edinburgh, Australia.

mailto:adrian.pincombe@dsto.defence.gov. au

2. B. M. Pincombe, Defence Science and Technology Organisation, Edinburgh, Australia. 